\title{
Bitcoin et chaîne de blocs : état des lieux et implications pour la gouvernance mondiale
}

\author{
Joanie Arsenault ${ }^{a}$, Myriam Ertz ${ }^{b}$
}

RÉSUMÉ. À la fin de l'année 2017, le cours du Bitcoin a frôlé la barre symbolique des 20000 dollars américains, créant ainsi un intérêt grandissant de la part des milieux d'affaires, des médias, des preneurs de décision, et du grand public. La communauté scientifique n'est pas en reste puisque des courants de recherche entiers sur le sujet sont apparus dans des disciplines aussi variées que la finance, l'économie, le marketing, l'éthique, l'informatique ou encore le droit. L'intérêt du duo cryptomonnaies - chaîne de blocs, en général, et du Bitcoin, en particulier -, s'est toutefois limité à l'examen des aspects techniques, des capacités transactionnelles et des implications pour le commerce et la finance. Très peu d'études se sont penchées sur l'examen des conséquences de ces systèmes d'échange décentralisés et pair-à-pair, tels que le Bitcoin et la chaîne de blocs, sur les configurations actuelles de la gouvernance mondiale. Cet article a pour objectif de faire un compte rendu commenté de l'ouvrage collectif Bitcoin and Beyond: Cryptocurrencies, Blockchains, and Global Governance. Dans cet ouvrage, Malcolm Campbell-Verduyn met à contribution plusieurs auteurs afin de mettre en lumière la manière dont la chaîne de blocs déborde du strict cadre économique et financier pour s'intégrer dans la gestion des sphères politique, légale et juridique. Ce faisant, l'ouvrage lève le voile sur de nombreuses implications des cryptomonnaies et de la chaîne de blocs pour la gouvernance mondiale, souvent méconnues et très peu étudiées dans la littérature, mais d’importance capitale dans un monde de plus en plus mondialisé.

\begin{abstract}
$\boldsymbol{A B S T R A C T}$. By the end of the year 2017, the stock price of the Bitcoin got close to the symbolic threshold of 20000 USD, which created a growing interest from business circles, media, decision-makers and the general public alike. The scientific community was not to be outdone since entire research streams emerged on this topic in various disciplines such as finance, economics, marketing, ethics, information technology, and laws. The interest for the cryptocurrencies - blockchain duo, in general, and Bitcoin, in particular-, was nonetheless limited to the study of technical aspects, transactional capacities, and implications for commerce and finance. So far, little research examined the consequences of decentralized, peer-to-peer exchange systems, such as Bitcoin and blockchain, on the current configurations of global governance. The objective of this article is to present a commentated report of the collective book. Bitcoin and Beyond: Cryptocurrencies, Blockechains, and Global Governance. In this book, Malcolm Campbell-Verduyn harnesses several authors in order to shed some light on the manner in which the blockechain goes beyond the strict economic and financial framework to enter the realm of politic, legal and judicial spheres. By so doing, the book. highlights numerous implications of cryptocurrencies and the blockchain for global governance, that are often unknown and not so much studied in the literature, but which lie at the crux of an increasingly globalized world.
\end{abstract}

\section{Introduction}

L'internationalisation des échanges financiers a amené son lot de défis importants liés à l'authentification des transactions. Afin de dissiper les incertitudes, de nouvelles institutions ont fait leur apparition, telles que le système interbancaire SWIFT, l'Organisation mondiale du commerce
(OMC), et les institutions financières pouvant utiliser SWIFT dans le cadre des transactions entre les pays et entre les devises (Godebarge et Rossat, 2016). L'idée d'incorporer une tierce partie de confiance (p. ex., SWIFT, OMC, institutions financières) entre deux échangeurs a amené une certaine sécurisation des transactions et une fluidité monétaire accrue entre les utilisateurs (Godebarge et Rossat, 2016). Toutefois, avec l'avènement de

\footnotetext{
a Étudiante au baccalauréat en administration des affaires, Université du Québec à Chicoutimi

b Professeure, Ph. D., Adm. A., Université du Québec à Chicoutimi
} 
multiples crises bancaires et, en particulier, la crise financière mondiale de 2008, ainsi que le risque systémique devenu inhérent à de nombreuses institutions bancaires, cette intermédiation a été peu à peu contestée par le grand public (De Bandt et al., 2015; Duport et al., 2018). En 2008, la publication d'un livre blanc rédigé par une personne ou un groupe de personnes dénommé "Satoshi Nakamoto » proposait une solution novatrice et radicale à cette problématique: l'indépendance pure et simple par rapport au système bancaire institutionnalisé (Humayun et Belk, 2018). À cette fin, le livre blanc préconisait le développement d'un système d'échanges financiers basé sur un réseau décentralisé, qui s'appellerait la « chaîne de blocs » ou blockchain, en anglais. L'existence d'une chaîne de blocs, cette infrastructure d'échange décentralisée, permet l'émergence d'un système financier parallèle au système financier conventionnel. Certains auteurs (p. ex., Humayun et Belk, 2018) parlent même d'« écosystème » afin de souligner l'aspect autocontenu et autoentretenu de ce nouveau système d'échanges financiers.

C'est dans ce nouveau cadre financier qu'émergea un type de monnaie jamais vu jusqu'alors, la cryptomonnaie. Appelée aussi «monnaie Internet» ou «monnaie virtuelle » (Inshyn et al., 2018), la cryptomonnaie a attiré un nombre grandissant d'investisseurs désireux de s'émanciper, sinon totalement, du moins partiellement, du réseau bancaire classique (Inshyn et al., 2018). Les cryptomonnaies ont de fait rapidement joui d'une grande popularité chez un nombre croissant d'investisseurs. Si certains travaux ont mis en évidence les facteurs contribuant à l'adoption des cryptomonnaies (p. ex., $\mathrm{Al}$ Shehhi et al., 2014), d'autres ont mis en évidence les freins à leur adoption, comme la résistance institutionnelle, les fluctuations de valeur des cryptomonnaies, la variété des cryptomonnaies, ou encore les risques liés aux fraudes transactionnelles (Arsenault et Ertz, 2018a, 2018b). Le coût énergétique immense lié aux analyses et aux transactions et, ce faisant, l'empreinte écologique importante des cryptomonnaies, pose également des défis d'envergure sur le plan environnemental (O'Dwyer et Malone, 2014). Pour l'heure, l'attrait des cryptomonnaies se situe davantage dans leur performance à titre d'outil d'investissement, plutôt que dans leur capacité d'agir en tant que moyen d'échange, c'està-dire de monnaie conventionnelle pour mener des transactions commerciales (Glaser et al., 2014).
Actuellement, la chaîne de blocs, sur laquelle circulent les nombreuses cryptomonnaies en usage, s'intègre de plus en plus dans un cadre de gouvernance mondiale. La notion de gouvernance peut être comprise de plusieurs manières. D'après Pierre (2000), il existerait cinq usages du concept de gouvernance dans les domaines du développement économique, des institutions internationales, de la gouvernance d'entreprises, des stratégies de management public et dans celui des nouvelles pratiques de coordination et de réseaux. Dans le cadre de l'administration publique, la gouvernance est définie comme «un changement du sens du terme gouvernement, référant à un nouveau processus de gouvernement, ou à des conditions transformées de réglementation, ou encore à de nouvelles méthodes par lesquelles la société est gouvernée » (Rhodes, 1997, p. 46). Elle met notamment l'accent sur les transmutations du rôle de l'État et notamment sur le fait que les mécanismes d'intervention publique centraux et hiérarchiques ont été remplacés par des mécanismes de marché dans les années 1980, et par le développement de réseaux dans les années 1990 (Enjolras, 2010). Les prérogatives croissantes aux niveaux supranationaux (p. ex., Union européenne, ALENA) et infranationaux (p. ex., régions en France, États/comtés aux ÉtatsUnis, communautés autonomes en Espagne, Länder en Allemagne) ont renforcé le passage d'une conception en matière d'administration publique (État) à une conception plus abstraite en matière de gouvernance (Enjolras, 2010).

Dans son ouvrage collectif Bitcoin and Beyond: Cryptocurrencies, Blockchains, and Global Governance, Malcolm Campbell-Verduyn ${ }^{1}$ met à contribution plusieurs auteurs afin de mettre en lumière la manière dont la chaîne de blocs déborde strictement du cadre financier pour s'intégrer dans la gestion des sphères politiques, légales et juridiques. De nombreux questionnements sont donc soulevés quant à l'implication de ces technologies au sein d'institutions centralisées, notamment au sein des gouvernements et des entreprises. Ils évaluent les implications des cryptomonnaies et de la chaîne de blocs pour la gouvernance mondiale contemporaine. Décrites comme décentralisées, les auteurs de l'ouvrage collectif évaluent la manière dont les cryptomonnaies et la chaîne de blocs induisent des formes de gouvernance à l'interne et à l'externe du système. Les contributions interdisciplinaires de cet ouvrage 
permettent d'établir et de fournir une compréhension du système de la chaîne de blocs et des changements engendrés par l'arrivée de cette technologie. L'ouvrage amène les lecteurs à discerner, tout d'abord, la structure logistique et enfin, les enjeux économiques, politiques et sociaux entourant ce phénomène. L'ouvrage met plus particulièrement en évidence, de manière inédite, les implications de la chaîne de blocs sur le plan de la gouvernance à l'échelle mondiale.

Cet article vise deux objectifs fondamentaux. Tout d'abord, il a pour but de présenter une introduction synthétique aux notions de chaîne de blocs et de monnaie virtuelle, c'est-à-dire des cryptomonnaies. En second lieu, il vise à présenter la pensée de Campbell-Verduyn ainsi que de ses collaborateurs sur les implications de la chaîne de blocs pour la gouvernance mondiale.

La structure de l'article comportera trois sections. Dans un premier temps, nous établirons une introduction sommaire aux monnaies virtuelles, c'est-àdire aux cryptomonnaies. En nous basant ensuite sur l'ouvrage de Campbell-Verduyn et de ses collaborateurs, nous introduirons plus en détail la notion de système de gouvernance à l'interne et à l'externe. Puis, l'article abordera le concept fondamental de "chaîne de blocs » afin de bien comprendre la technologie à l'étude et ses implications. La troisième partie sera consacrée aux implications de la chaîne de blocs pour la gouvernance mondiale.

\section{Introduction aux monnaies virtuelles}

En 2008, au moment même où une crise financière importante s'abat sur l'économie mondiale, une cryptomonnaie, nommée "Bitcoin», fait son apparition (Stiglitz, 2010). Sous le pseudonyme de Satoshi Nakamoto, une synthèse de neuf pages explique les fonctionnalités et les motivations derrière la cryptomonnaie et son réseau, la chaîne de blocs. L'arrivée de la cryptomonnaie fait un pied de nez au système bancaire traditionnel et profite du contexte économique défavorable pour gagner la confiance d'investisseurs de plus en plus sceptiques envers les institutions financières classiques (Corradi et Höfner, 2018). En effet, l'effondrement de plusieurs institutions financières ébranle la crédibilité des banques et les gouvernements seront critiqués pour le sauvetage à même les fonds publics du système financier ainsi que pour les politiques monétaires « accommodantes » des diverses banques centrales (Braun, 2016). Eu égard aux politiques monétaires accommodantes, les critiques portent particulièrement sur les multiples assouplissements quantitatifs, consistant en des achats massifs d'obligations (c'est-à-dire des titres de créances émis par des gouvernements ou des organisations) par les banques centrales, et les taux d'intérêt négatifs ainsi engendrés pour les déposants (Corradi et Höfner, 2018).

Bien que la crise financière fût un moment opportun pour l'arrivée des cryptomonnaies, de nombreux incidents sont venus ternir la réputation de ce système supposément incorruptible.

Un premier scandale surgit avec la couverture médiatique visant Silk Road, un marché noir en ligne ayant pour caractéristique d'utiliser le réseau Tor afin d'assurer l'anonymat des acheteurs et vendeurs de produits controversés, voire illégaux, tels que des drogues. Cette infrastructure électronique d'échange accepte en exclusivité la cryptomonnaie Bitcoin, qui permet alors un grand nombre d'échanges entre des intermédiaires anonymes. À la manière d'un «eBay du crime », Silk Road donnait accès à « un choix de plus de treize mille $[s i c]$ médicaments, des services de piratage informatique, des logiciels malveillants, de faux passeports, de fausses cartes de crédit et même, selon le FBI, des services de tueur à gages » (Musiani et al., 2018, p. 145). Selon un acte d'accusation, 15000 utilisateurs distincts utilisaient cette plateforme de vente. Son propriétaire, Ross William Ulbricht, sera par la suite emprisonné sans cesser de promouvoir le modèle libertaire et une économie exempte de toute intervention de l'État. À la suite de son arrestation, les autorités procédèrent à l'identification des acheteurs et des vendeurs afin de faire cesser toute activité commerciale non réglementaire transigeant par Silk Road.

Le deuxième scandale associé à l'émergence des cryptomonnaies est relié à la fermeture de la plateforme $M t G o x$, créée en 2007, mise sur pied par Jed McCaleb et prise en charge ultérieurement par divers gestionnaires. Semblable à Silk Road, MtGox joua un rôle important à titre d'intermédiaire dans le marché de l'achat et de la vente du Bitcoin. De son 
ascension à sa chute, les auteurs relèvent quatre éléments qui ont conduit la plateforme à sa fermeture. Tout d'abord, MtGox fut de nombreuses fois l'intermédiaire d'échanges massifs de devises étrangères, soutenant ainsi une importante circulation monétaire illégale. Ensuite, le fort volume de transactions fut affecté par de nombreuses défaillances techniques, le ralentissement puis la paralysie totale, et la perte de crédibilité chez ses utilisateurs. Un troisième élément fut caractérisé par « des retards dans l'exécution des retraits d'argent, perturbation causée par le flux important et le prix des devises » (Musiani et al., 2017, p. 147). Enfin, le dernier élément fut la démission de Mark Karpelès de la Fondation Bitcoin (Southhurst, 2014), dernier directeur de la plateforme et reconnu maintenant comme criminel. Ces événements fortement médiatisés propulsèrent les cryptomonnaies, en général, et le Bitcoin, en particulier, sur le devant de la scène mondiale.

Un troisième scandale ayant contribué à la notoriété des cryptomonnaies survint en mars 2013. Une série d'événements reliés à une défaillance dans un protocole de minage - validation des transactions passant par la chaine de blocs - engendra, une fois de plus, la perte de confiance des utilisateurs. Les auteurs relèvent notamment que :

Entre le 11 et le 12 mars 2013, un mineur utilisant la version $0.8 \mathrm{du}$ protocole Bitcoin accepta un grand bloc de transactions non valides. Dans les limites du logiciel, cela entraîna une division involontaire de la Blockchain Bitcoin. Cette scission entraîna donc la création de deux registres divergents sans consensus clair, ni même la reconnaissance entre les deux registres. Les mineurs ont ensuite résolu la scission en rétrogradant le protocole à la version 0.7 et en soutenant uniquement l'un des deux registres (Musiani et al., 2017, p. 139).

\subsection{Bitcoin : monnaie emblématique}

Monnaie emblématique de la mouvance des cryptomonnaies, les Bitcoins s'échangent sur un réseau pair-à-pair, nommé la Bitcoin Blockchain. Le Bitcoin est limité à 21 millions de jetons pour deux grandes raisons. Tout d'abord, l'instauration d'une limite est un vecteur de stabilité. Lorsque la limite des 21 millions d'unités sera atteinte, le Bitcoin sera moins spéculatif et moins sujet à une volatilité extrême. En second lieu, la limitation des Bitcoins donne de la valeur, car la rareté contribue à la fixation du prix d'un bien. C'est notamment le cas pour les métaux précieux tels que l'or ou le platine, lesquels ont un prix élevé du fait de leur rareté. Le même effet a été délibérément recherché pour le Bitcoin.

Chaque bloc de transactions, contenant les jetons numériques que sont les Bitcoins, est initialement fixé à un mégaoctet et contient à lui seul plusieurs transactions. Une fois le bloc formé, celui-ci sera émis au sein de la chaîne de blocs afin d'effectuer le transfert entre deux utilisateurs, d'où l'origine du nom de ce réseau "pair-à-pair». À l'instar des autres cryptomonnaies, le Bitcoin est donc intégré dans une communauté numérique où de nombreuses transactions sont relayées par l'entremise de la chaine de blocs, laquelle effectuera donc le pont entre les parties impliquées dans l'échange. En ce qui a trait à la validation des transactions, ce sont les mineurs qui veillent à la vérification de la signature électronique, de l'adresse de l'expéditeur ainsi qu'à la possession de fonds. Ce capital humain, travaillant au sein du cyberespace, n'est pas affilié à une quelconque institution. Puisque le réseau est décentralisé, toute personne ayant un équipement pour être mineur détient la possibilité de le devenir. Par contre, puisque le registre est basé sur une comptabilité compétitive, les mineurs doivent être dotés d'infrastructures informatiques performantes afin de se joindre à la communauté et réaliser une rémunération.

\subsection{La gouvernance interne et externe des cryptomonnaies}

L'ouvrage de Campbell-Verduyn permet de distinguer en tout premier lieu les types de gouvernance interne et externe reliés aux cryptomonnaies. La gouvernance interne fait référence aux prises de décision et à la gestion ayant trait à la conception ainsi qu'aux aspects techniques d'une cryptomonnaie en particulier (p. ex., Ethereum). Quant à la gouvernance externe, elle est décrite comme « l'influence exercée par la communauté, les médias et le grand public » (Campbell-Verduyn, 2017, p. 23) sur les différentes cryptomonnaies.

L'auteur distingue ici trois formes de gouvernance interne. Premièrement, les mineurs constituent un pouvoir de décision au sein de leur chaîne de blocs puisqu'ils détiennent le pouvoir 
de valider ou non une transaction. Autrement dit, le réseau permet aux mineurs de converger rapidement vers d'autres transactions qui leur paraissent plus fiables.

Deuxièmement, les programmeurs derrière les applications de chaîne de blocs « constituent également des acteurs clés puisqu'ils sont les architectes de la plateforme » (Campbell-Verduyn, 2017, p. 23). Cependant, certaines organisations exigent un consensus des mineurs avant d'effectuer des modifications au sein de l'application chaîne de blocs. Ces consensus s'établissent dans le cadre de forums ou de votes formels, afin de conserver l'aspect décentralisé, pair-à-pair, et collégial du réseau.

Troisièmement, il existe une forme de financement privilégiant certaines devises numériques; c'est le cas du marché de change et intermédiaire d'échanges, Ripple. Au même titre que les autres cryptomonnaies, Ripple est soutenu par du capital risque ainsi que par de nombreuses fonctions commerciales autant en ce qui concerne le développement des affaires, du marketing, du financement, que la conception de nouveaux produits, c'est-à-dire de nouvelles monnaies numériques notamment (Campbell-Verduyn, 2017).

Campbell-Verduyn estime que le niveau d'implication externe de la gouvernance est moindre comparativement à l'interne puisque, la plupart du temps, les réactions à l'externe se résument généralement à des réactions aux défaillances observées à l'interne (Walsh et Seward, 1990). En clair, la gouvernance externe ne s'active que lorsque la gouvernance interne fait défaut. De plus, comme la chaîne de blocs est caractérisée par une programmation rigide, il est très difficile pour les acteurs externes d'exercer une quelconque influence sur le registre. Le grand public, la communauté et les médias ne détiennent que très peu de pouvoir sur la gestion et l'organisation des cryptomonnaies. La faible maitrise des connaissances techniques qu'exige la compréhension des mécanismes soustendant la chaîne de blocs et les cryptomonnaies, constitue une autre raison importante expliquant le peu de prise qu'a la gouvernance externe sur la chaîne de blocs, en général, et sur les cryptomonnaies, en particulier. Ce point est d'ailleurs dénoncé par les acteurs clés de la gouvernance externe, soit le public, la communauté, et les médias. En réalité, au sein du public et de la communauté, CampbellVerduyn présente l'État comme le seul acteur externe réellement pertinent. Par son exercice des pouvoirs législatifs, judiciaires, de la violence légitime, et du droit de battre monnaie, les gouvernements détiennent a priori un pouvoir dans l'encadrement des cryptomonnaies. Pensons notamment à l'Autorité des marchés financiers du Québec, au Canada, ou encore à la Banque de France, avisant les investisseurs et les émetteurs des nombreux risques associés à l'achat ou à la vente de cryptomonnaies. Cette pression étatique sur les acteurs présente potentiellement la seule forme de pouvoir qui puisse représenter un frein majeur à l'adoption des cryptomonnaies dans l'économie actuelle.

\section{Les chaînes de blocs : une innovation dans l'architecture fondamentale des échanges monétaires}

Au cours de l'Histoire, le pouvoir de battre monnaie a généralement été l'apanage des autorités publiques, c'est-à-dire d'une autorité centrale ayant souvent un contrôle relativement total sur l'émission de ladite monnaie. Si des solutions locales étaient parfois développées entre individus de manière décentralisée, ces systèmes d'échange s'effectuaient généralement à très petite échelle et dans des systèmes socialement et géographiquement très limités. Avec l'avènement des technologies numériques et du mouvement pair-à-pair (peer-to-peer) ayant accompagné cette évolution majeure, la monnaie - au même titre que les CD, les DVD, et les jeux vidéo - s'est virtualisée pour s'échanger à l'intérieur d'un réseau décentralisé pair-à-pair. La chaîne de blocs est ainsi devenue à la monnaie et aux institutions financières, ce que Napster ou Kazaa sont devenus pour le $\mathrm{CD}$ et l'industrie musicale.

Il existe quatre types de registres de chaine de blocs, pouvant être "classés en fonction du mode d'accessibilité et de lecture des nœuds, à savoir "public» ou "privé»; ainsi qu'en fonction du mode d'écriture dans le registre, à savoir, «avec permission » et «sans permission» (Ghilal et Nach, à paraître) (voir le tableau 1). 


\begin{tabular}{|l|l|l|}
\hline $\begin{array}{l}\text { TYPES DE } \\
\text { CHAÎNES } \\
\text { DE BLOCS }\end{array}$ & \multicolumn{1}{|c|}{ Avec permission } & \multicolumn{1}{c|}{ Sans permission } \\
\hline Public & $\begin{array}{l}\text { Aucune restriction sur la lecture des don- } \\
\text { nées du réseau, mais l'ajout des transac- } \\
\text { tions est réservé à une liste prédéfinie } \\
\text { d'intervenants ayant des identités connues. }\end{array}$ & $\begin{array}{l}\text { Aucune restriction sur la lecture des don- } \\
\text { nées et la soumission des transactions à } \\
\text { inclure dans le réseau. C'est un réseau } \\
\text { distribué sous forme de protocoles } \\
\text { publics basés sur des algorithmes de } \\
\text { consensus (preuve de travail). }\end{array}$ \\
\hline \multirow{2}{*}{$\begin{array}{l}\text { Privé } \\
\text { Exemple : Ethereum }\end{array}$} & $\begin{array}{l}\text { Configuration dans laquelle l'accès direct } \\
\text { aux données de la chaîne de blocs et la } \\
\text { soumission des transactions sont limités } \\
\text { à une liste prédéfinie d'entités. }\end{array}$ & $\begin{array}{l}\text { Cette configuration est peu fréquente, car } \\
\text { selon la perspective d'ouverture et de } \\
\text { transparence promue au sein de la chaîne } \\
\text { de blocs, les modes de lecture et d'écriture } \\
\text { devraient a priori être attribués à toutes les } \\
\text { entités du réseau privé. }\end{array}$ \\
& $\begin{array}{l}\text { Exemple : Ripple } \\
\text { Exemple : S.O. }\end{array}$ \\
\hline
\end{tabular}

Tableau 1 - Typologie des chaînes de blocs

Source : Ghilal et Nach (à paraitre)

Dans la conclusion de son mémorandum, Nakamoto discute de la fiabilité des nœuds au sein de l'application, en raison de sa simplicité et de sa fonction décentralisée. Abordant le problème de la double dépense, soit le risque de dépenser deux fois une valeur monétaire au sein du réseau, il propose le réseau pair-à-pair afin de bâtir un registre transactionnel honnête des opérations effectuées. Afin de valider les opérations, il suggère le mécanisme du consensus, soit la preuve de travail détenue par les mineurs. Leur travail consiste à accepter ou décliner toutes transactions au sein du système. (Nakamoto, 2008) Rapidement reprise par une communauté de soutien anonyme, la configuration de la chaine de blocs a évolué de manière à permettre plusieurs types de transactions. Parmi cellesci figurent des " opérations commerciales ou boursières, des contrats, des accords, ou encore, en informatique, des opérations élémentaires de saisie ou de consultation d'information » (Godebarge et Rossat, 2016, p. 1).

En outre, la chaîne de blocs détient le double avantage de l'anonymat et de la traçabilité grâce à son registre transactionnel transparent. Cela constituerait donc une solution « à la surveillance généralisée du gouvernement et des entreprises telle que révélée par Edward Snowden» (Campbell-Verduyn,
2017 , p. 2), un ancien employé de la NSA et de la CLA. L'application permet également de parer aux instabilités financières, notamment dans la zone euro, toujours relativement fébrile autant sur le plan économique que financier. Cela est notamment devenu apparent lors de « la confiscation des dépôts dans le renflouement des banques chypriotes » (Campbell-Verduyn, 2017, p. 2). La chaîne de blocs apparaît alors comme un moyen pour les citoyens, les investisseurs institutionnels (p. ex., sociétés d'assurance, caisses de retraite) et privés (p. ex., fonds d'investissement des banques et caisses, entreprises privées), voire les gouvernements (p. ex., Venezuela, Turquie, Iran), de se soustraire à des activités de spéculation effectuées sur leurs devises nationales.

D'un point de vue fonctionnel, la technologie derrière la chaîne de blocs consiste en une plateforme numérique agissant comme intermédiaire dans le mouvement des cryptomonnaies entre deux parties et ce, sans tierce partie (absence de tout « garant de confiance »). Les utilisateurs doivent avoir accès à un réseau Internet libre et donc universel afin de pouvoir effectuer leurs transactions. Globalement, un réseau comporte un ensemble de plusieurs procédés technologiques établis. Nous retrouvons le 
cryptage numérique servant à masquer partiellement ou complètement le contenu d'une transaction. Ensuite, s'ajoutent à cela de puissants algorithmes mathématiques permettant de résoudre les équations préalables au consensus validant la transaction. Comme le démontre la figure 2, cette étape est rendue possible grâce aux ordinateurs et est effectuée par les ressources informatiques fournies par les mineurs. Enfin, nous retrouvons aussi l'horodatage qui consiste en un principe d'empreinte. Un serveur réunit tout d'abord un ensemble d'objets (transactions) et prend l'empreinte (hash) de cet ensemble. Ensuite, il annonce cette empreinte sous la forme d'un message sur un forum Usenet, soit le système de réseaux de forums. Enfin, tous les horodatages conservent l'horodatage précédent, ce qui constitue la chaîne (Godebarge et Rossat, 2016). En ce qui a trait à l'accessibilité sur le réseau, le concept des transactions repose sur la cryptographie asymétrique. Elle permet de valider les informations relatives à une transaction en chiffrant les données reliées et son origine (Godebarge et Rossat, 2016).

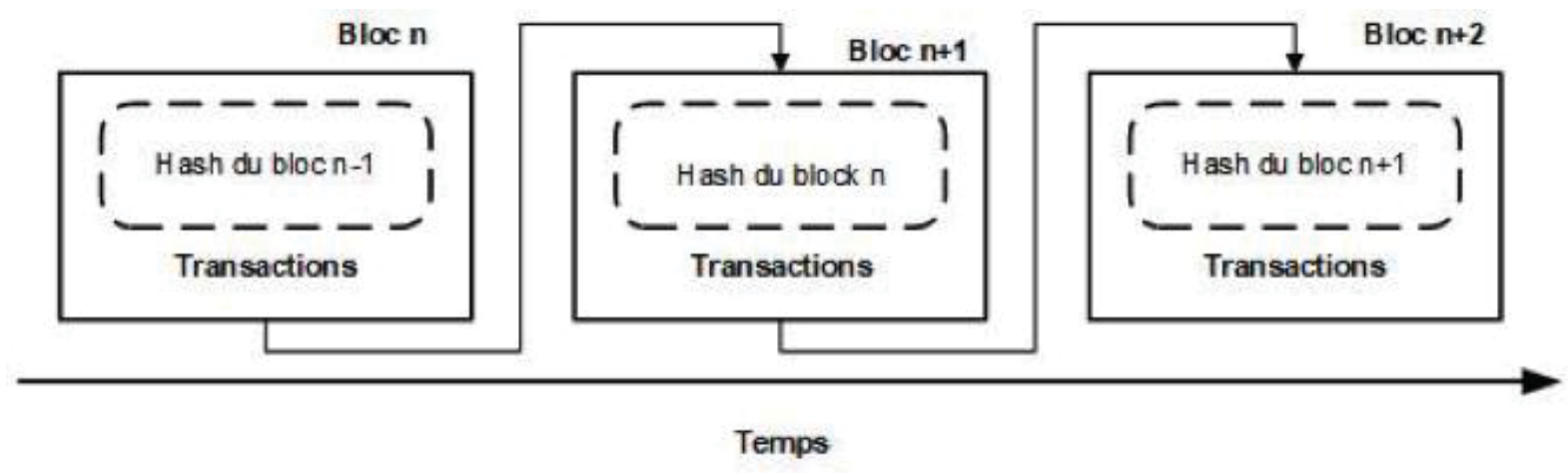

Figure 1 - Vue générique de la chaîne de blocs Source : Christidis et Devetsikiotis (2016)

Il y a une clé publique, celle-ci représente un identifiant consigné dans un registre accessible à tous. Quant à la clé privée, elle est détenue uniquement par son utilisateur et représente son seul moyen d'accéder à la base de données.

La caractéristique première de la chaîne de blocs réside donc dans le fait qu'aucune entité ou aucun ordinateur n'est responsable du registre transactionnel. Considérant qu'aucune autorité centrale ne contrôle les transactions, le système fonctionne par l'entremise d'un large réseau de mineurs, où son système de validation sera nommé la preuve de travail. Les mineurs font donc référence au capital humain et matériel approuvant l'intégrité des transactions. À la suite de la conception, les transactions seront reliées entre elles séquentiellement et prêtes pour leur envoi au sein du système. Cette technologie nécessite donc la maintenance d'un audit continu pour répertorier tous les changements effectués au sein de la base de données. Les cryptomonnaies sont souvent décriées pour leur manque de contrepartie en matière de valeur. Or, le capital humain et les ressources importantes pour le fonctionnement de ce système peuvent être considérés comme les contreparties sources de valeur aux cryptomonnaies.

Bien que la chaîne de blocs soit principalement utilisée pour l'échange de cryptomonnaies, différentes utilisations additionnelles sont possibles avec cette technologie émergente. Ainsi, des entreprises et des gouvernements ont su profiter des avantages de la chaîne de blocs afin d'optimiser leurs processus de gestion de la qualité et de l'approvisionnement. Par exemple, la multinationale Walmart profite des avantages de la chaîne de blocs pour accéder à son inventaire de produits en temps réel, et ce, en protégeant l'entreprise des vols de données et du piratage informatique (Bloomberg, 2018).

\section{Perspectives dans la gouvernance comtemporaine mondiale}

L'examen de la chaîne de blocs met en lumière plusieurs éléments de la gouvernance contemporaine mondiale du XXIe siècle. L'intégration de ces technologies signalerait l'émergence d'une tendance de 
fond vers la décentralisation et le démantèlement graduel de grandes institutions. Cela avait également été mis en lumière par Davis en 2013 dans son article After the Corporation, dans lequel il stipulait que le démantèlement des grandes corporations centralisées en unités d'affaires stratégiques flexibilisées (philosophie lean et agile), relocalisées et ayant un recours croissant à la sous-traitance, au travail autonome et contractuel, se reflétait sur le plan politique avec le retrait graduel d'un État central et fort d'un nombre croissant de domaines (p. ex., privatisations, libéralisation, dérégulations) (Enjolras, 2010). Cette décentralisation institutionnelle se doublera d'un pendant virtuel, c'est-à-dire que la décentralisation des institutions s'effectuera largement dans le cadre du numérique, grâce notamment à la chaîne de blocs.

L'attrait de la technologie réside dans le fait que la confiance en l'échange direct entre pairs - exempte de l'implication d'une autorité centrale - transcende la confiance dans des institutions centralisatrices et établies, mais de plus en plus critiquées. Ici, il importe de noter qu'il ne s'agit pas d'une position partisane et essentialiste, où centralisation équivaut au mal et décentralisation équivaut au bien, mais plutôt de la constatation d'un état de fait et d'une évolution dans les configurations de pouvoir. Alors que les institutions religieuses - en particulier l'Église catholique - voient leur influence diminuer depuis le XIX siècle, ce sont à présent les gouvernements qui font face à la défiance des citoyens et des votants qui ont perdu confiance dans la classe politique (Lipovetsky, 2003). L'élection de l'antipoliticien Donald Trump aux commandes de la première puissance économique ainsi que le Brexit sont autant de signes de cette défiance en émergence en Occident. Les regroupements supranationaux centralisateurs de type technocratique tels que l'Union européenne sont également sujets à un rejet croissant (p. ex., le «non» aux référendums français et néerlandais, Brexit de 2016, mouvements populistes, souverainistes, nationalistes et eurosceptiques en Europe). Les grandes institutions financières combinant les activités de dépôt et d'affaires (p. ex., banques) font face à la critique populaire depuis la crise financière mondiale de 2008 en particulier (p. ex., Occupy Wall Street aux États-Unis, parti politique Podemos en Espagne) (Ertz, Durif et Arcand, 2017). Enfin, les grandes centrales syndicales ne sont plus perçues comme étant utiles et efficaces pour la défense des intérêts des travailleurs (Lipovetsky, 2003). Sur le plan économique, les applications et plateformes d'échange de tous types de biens et services de pair-à-pair, dénommées sous l'oxymore économie du partage ou économie collaborative, constituent un autre exemple de la recherche de solutions de rechange aux grands acteurs économiques établis (Botsman et Rogers, 2010). L'importance de la chaîne de blocs est ainsi plus aisément compréhensible à la lumière de cette tendance de fond à la décentralisation, laquelle est visible dans les sphères économique, politique, financière et sociale.

L'aspect technologique de la chaîne de blocs et des cryptomonnaies a masqué leurs implications plus larges pour la gouvernance mondiale. Si ses applications politicolégales ont été, pour l'heure, relativement ignorées, certains auteurs, notamment Campbell-Verduyn et ses collaborateurs, se sont néanmoins interrogés sur la capacité de cet ensemble technologique à pallier certains problèmes de gouvernance. Les auteurs se sont également demandé s'il était souhaitable que cette technologie s'applique à tous les acteurs économiques et politiques mondiaux. De nombreuses questions furent soulevées que l'on peut résumer en trois points clés.

Tout d'abord, les auteurs se demandent si la chaine de blocs n'entrainerait tout simplement pas de nouveaux problèmes de gestion, cette application posant alors plus de problèmes qu'elle n'en résoudrait. D'après eux, la gestion par chaîne de blocs pourrait renforcer le pouvoir de certains acteurs face à d'autres. Il y aurait un certain manque de transparence de certains agents au sein même de l'institution et de son application par la chaîne de blocs. L'auteur soulève, par exemple, que « différents programmeurs détenant des compétences leur permettant de naviguer dans la complexité technique et d'exercer ce pouvoir en devenant des «initiés-clés » peuvent altérer la forme de gouvernance décentralisée promue par l'utilisation de la chaîne de blocs » (Campbell-Verduyn, 2018, p. 182). La décentralisation utopiste ne serait donc qu'une croyance naïve. Pour renforcer ce point, Campbell-Verduyn parle de «micro-hiérarchie et d'asymétrie de pouvoir, c'est-à-dire de petites alliances stratégiques, au sein d'une institution. Ces associations peuvent provenir de rassemblements entre les développeurs, les intermédiaires et le bassin de mineurs » (CampbellVerduyn, 2018, p. 183). En clair, il ne s'agirait ni 
plus ni moins de la configuration d'un nouveau système au complet, plutôt que d'un système grassroots, alternatif, critique au système dominant.

En second lieu, les auteurs se questionnent sur la prise de position des différents agents et leur satisfaction eu égard à la technologie de la chaîne de blocs. En clair, comment se polarisent les attitudes des agents envers la chaîne de blocs? Sont-ils globalement en faveur ou en défaveur de la technologie? Pourquoi? À cela, les auteurs répondent que les bénéfices rattachés à l'application de la chaîne de blocs varient en fonction de l'institution. Pour les gouvernements, le Bitcoin, en particulier, demeure l'archétype de controverses auxquelles ils ne savent pas réellement répondre. Toutefois, la chaine de blocs serait plus acceptable en raison du progrès technique indéniable qu'elle apporte ainsi que de son utilité potentielle. Les gouvernements européens et américains semblent afficher une tendance à l'acceptation de ce modèle numérique. Le raisonnement est le suivant : "l'application de règlementations [sic] très strictes risquerait de conduire rapidement l'activité Bitcoin ailleurs, affaiblissant ainsi l'emprise des régulateurs sur les institutions et les intermédiaires se développant dans cet écosystème » (Böhme et al., 2015). Les gouvernements sont donc réticents à une acceptation totale de la chaîne de blocs, mais craignent néanmoins que des lois trop sévères éloignent la technologie hors de leur champ d'action (Böhme et al., 2015). Certains autres bénéfices économiques ont été avancés au sujet de la chaîne de blocs. Les entreprises en démarrage peuvent bénéficier de l'utilisation de l'application chaine de blocs et de sa transparence pour devenir plus autonomes et remplacer les anciennes formes de gestion au sein de l'entreprise.

Troisièmement, les auteurs se demandent si ce système surmonte réellement les difficultés actuelles. À cet effet, certains suggèrent que «les technologies nouvelles et émergentes furent largement abordées et favoriseraient la coopération et les progrès soulignés dans les récits libéraux et plus technoutopiques, mais aussi comme donnant naissance à des formes technocratiques de gouvernement, ces dernières étant caractérisées par les inégalités et les asymétries de pouvoir soulignées dans des perspectives critiques et plus technodystopiques » (Campbell-Verduyn, 2017, p. 4). En somme, pour l'heure subsiste une vision utopiste et naïve de l'application de la technologie de la chaîne de blocs à la gouvernance globale. D'après les auteurs, cette application aboutirait rapidement à une bataille de pouvoir entre les intervenants et ultimement à la mise en place d'un pouvoir de type technocratique - probablement lié à la maitrise informatique et technique - plutôt que démocratique, par le peuple et pour l'intérêt collectif. Ainsi plutôt qu'un outil «antisystème », le complexe technologicofinancier de la chaîne de blocs et des cryptomonnaies ne serait que l'avatar d'un nouveau système de gouvernance autrement plus asymétrique et invasif que les systèmes actuels qu’il prétend ou est censé combattre.

\section{Le futur}

Pour Campbell-Verduyn, il demeure encore difficile de prédire les applications et l'évolution des technologies émergentes dans le cadre de la gouvernance mondiale contemporaine. Soulignant que la technologie de la chaîne de blocs s'appuie fondamentalement sur un Internet libre, une telle technologie apporte son lot de défis, notamment les perturbations engendrées par l'augmentation et l'hétérogénéité des cryptomonnaies. Bien qu'une concurrence saine des cryptomonnaies soit souhaitable, elle ne l'est pas nécessairement dans certaines sphères d'activités sociales à l'échelle mondiale, notamment sur le plan de la gouvernance. En effet, leur diversité pourrait amener une variété de nouvelles formes de gouvernance ou des niveaux de gouvernance très disparates. Alors que la notion de gouvernance souffre déjà d'une polysémie certaine sans les cryptomonnaies, la polyphonie conceptuelle ne sera que renforcée par une multiplicité de gouvernances associées à diverses cryptomonnaies.

Enfin, une tendance à la décentralisation politique, voire une perte du pouvoir centralisé, pourrait créer une forme de concurrence relative au monopole sur les monnaies nationales par la dénationalisation monétaire. En clair, les monnaies nationales, dont une grande partie est encore en circulation sous forme physique avec les pièces et les billets, pourraient disparaitre et être remplacées par les cryptomonnaies par essence exclusivement virtuelles. Le contrôle de la monnaie en serait grandement facilité, mais cette facilité vient avec un danger accru sur le plan de la gouvernance. Quid de l'identité de l'entité ou des entités responsables du contrôle de ces flux massifs de monnaies virtuelles? À cela s'ajoute l'absence de recours évoquant le sentiment 
d'injustice chez les spécialistes de l'éthique des affaires (Dierksmeier et Steele, 2016). Parallèlement, l'auteur avance que la chaîne de blocs pourrait servir à renforcer la centralisation de certaines gouvernances, en utilisant à bon escient ces technologies émergentes. Aux Pays-Bas, l'État a impliqué la chaine de blocs pour retrouver des acteurs participant à des crimes tels que le blanchiment d'argent - dont le Bitcoin -, la corruption et des abus (Bohannon, 2016). Mais a priori, cette technologie pourrait également être utilisée pour retracer tous types de transferts monétaires, ce qui pose des problématiques éthiques quant au respect de la vie privée.
Pour conclure, cet article présente une approche globale de la technologie de la chaîne de blocs et des cryptomonnaies, basée sur l'ouvrage collectif de Campbell-Verduyn et de ses contributeurs. Les auteurs ont soulevé la question de la gouvernance globale et de la décentralisation des systèmes, de manière à évaluer l'implantation mondiale sur les plans gouvernemental et financier des cryptomonnaies et de la chaîne de blocs. Devant la complexité de ces systèmes et des enjeux actuels, les lecteurs trouveront un grand intérêt à prendre connaissance de cet ouvrage afin de pouvoir comprendre l'implication normative des technologies émergentes de la chaine de blocs et des cryptomonnaies au sein des gouvernements et des entreprises multinationales.

\section{NOTES}

1 Malcolm Campbell-Verduyn est un politologue canadien dont les intérêts de recherche portent sur l'économie et la politique internationale. Actuellement professeur adjoint à l'Université de Toronto, il a publié de nombreux articles sur l'évolution financière mondiale reliée à la gouvernance et à de nouveaux systèmes, tels que le Bitcoin. Il a notamment effectué des travaux de recherche sur le poids des crises financières, et la légitimation de la chaîne de blocs principalement au sein de l'Union européenne.

\section{RÉFÉRENCES}

Al Shehhi, A., Oudah, M. et Aung, Z. (2014). Investigating factors behind choosing a cryptocurrency. Dans Industrial Engineering and Engineering Management (IEEM) (pp. 1443-1447). 2014 IEEE International Conference on IEEE.

Arsenault, J. et Ertz, M. (2018a). An exploratory study of cryptocurrencies usage in current exchange. Communication présentée à la $1^{\text {re }}$ FinteQC Conference, Lévis. Québec.

Arsenault, J. et Ertz, M. (2018b). Factors hindering the adoption of cryptocurrencies in commercial transactions: A topological and vector psychology perspective. Communication présentée à la Digital, Innovation \& Financing (DIF) Conference, Lyon, France.

Bloomberg (2018). Walmart is getting suppliers to put food on the blockchain. Repéré à https://www.bloomberg.com/news/articles/2018-04-23/walmart-is-getting-suppliers-to-put-food-on-blockchain-to-track

Bohannon, J. (2016). The Bitcoin busts. Science, 351(6278), 1144-1146.

Böhme, R., Christin, N., Edelman, B. et Moore, T. (2015). Bitcoin: Economics, technology and governance. Journal of Economic Perspectives, 29(2), 213-238.

Botsman, R. et Rogers, R. (2011). What's mine is yours: How collaborative consumption is changing the way we live. New York, NY: Penguin Press.

Braun, B. (2016). Speaking to the people? Money, trust and central bank legitimacy in the age of quantitative easing. Review of International Political Economy, 23(6), 1064-1092.

Campbell-Verduyn, M. (dir.) (2018). Bitcoin and beyond: Cryptocurrencies block chains, and global governance. Abingdon, Royaume-Uni: Routledge.

Christidis, K. et Devetsikiotis, M. (2016). Blockchains and smart contracts for the internet of things. IEEE Access, 4, $2292-2303$.

Corradi, F. et Höfner, P. (2018). The disenchantment of Bitcoin: Unveiling the myth of a digital currency. International Review of Sociology, 28(1), 193-207.

Davis, G. F. (2013). After the corporation. Politics \& Society, 41(2), 283-308.

De Bandt, O., Héam, J. C., Labonne, C. et Tavolaro, S. (2015). La mesure du risque systémique après la crise financière. Revue économique, 66(3), 481-500.

Dierksmeier, C. et Steele, P. (2016). Cryptocurrencies and business ethics. Journal of Business Ethics, 1-14. 
Duport, N., Fina, É. et Goyeau, D. (2018). Diversification des institutions financières et risque systémique : la prise en compte des risques extrêmes. Revue Économique, 69(3), 477-504.

Enjolras, B. (2010). Gouvernance verticale, gouvernance horizontale et économie sociale et solidaire : le cas des services à la personne. Géographie, Économie, Société, 12(1), 15-30.

Ertz, M., Durif, F. et Arcand, M. (2016). An analysis of the origins of collaborative consumption and its implications for marketing. Academy of Marketing Studies Journal, 21(1), 1-17.

Ghilal, R. et Nach, H. (à paraître). La technologie de la chaîne de blocs : fondements et applications. Dans M. Ertz, D. Hallegatte et J. Bousquet (dir.), Les reconfigurations de l'échange marchand. Québec, Québec : Presses de l’Université du Québec.

Glaser, F., Zimmermann, K., Haferkorn, M., Weber, M. C. et Siering, M. (2014). Bitcoin - asset or currency? Revealing users' bidden intentions. London, Royaume-Uni: ECIS.

Godebarge, F. et Rossat, R. (2016). Principes clés d'une application Blockchain. EM Lyon Business School. Repéré à https://www.coursehero.com/file/29197828/GODEBARGE-ROSSAT-Blockchain-version-finalepdf/

Humayun, M. et Belk, R. W. (2018). "Satoshi is dead. Long live Satoshi”: The curious case of Bitcoin's creator. Dans S. N. N. Cross, C. Ruvalcaba, A. Venkatesh et R. W. Belk (dir.), Consumer culture theory (Research in consume behavior volume 19) (p. 19-35). New York, NY: Emerald Publishing Limited.

Inshyn, M., Mohilevskyi, L. et Drozd, O. (2018). The issue of cryptocurrency legal regulation in Ukraine and all over the world: A comparative analysis. Baltic Journal of Economic Studies, 4(1), 169-174.

Lipovetsky, G. (2003). La société d'hyperconsommation. Le débat, 2, 74-98.

Musiani, F., Mallard, A. et Méadel, C. (2018). Governing what wasn't meant to be governed: A controversy-based approach to the study of Bitcoin governance. Dans M. Campbell-Verduyn (dir.). Bitcoin and beyond: Cryptocurrencies, block chains, and global governance (p. 133-156). Abingdon, Royaume-Uni: Routledge.

Nakamoto, S. (2008). Bitcoin: A peer-to-peer electronic cash system. Repéré à https://bitcoin.org/bitcoin.pdf

O'Dwyer, K. J. et Malone, D. (2014, juin). Bitcoin mining and its energy footprint. Dans les actes du congrès IET Irish Signals \& Systems Conference, Limerick, Irlande, Repéré à http://digital-library.theiet.org/content/conferences/10.1049/cp.2014.0699

Pierre J. (dir.) 2000. Debating governance. Oxford, Royaume-Uni: Oxford University Press.

Rhodes, R. A. (1997). Understanding governance: Policy networks, governance, reflexivity and accountability. London, Royaume-Uni: Open University Press.

Southhurst, J. (2014, février). MtGox CEO Mark Karpelès resigns from Bitcoin Foundation Board. Coindesk. Repéré à www.coindesk.com/mt-gox-ceo-mark-karpeles-resigns-bitcoin-foundation-board/

Stiglitz, E. J. (2010). Freefall. America, free markets and the sinking of world economy. New York, NY: Norton.

Walsh, J. et Seward, J. (1990). On the efficiency of internal and external corporate control mechanisms. Academy of Management Review, 15(3), 421-425. 